\title{
Estimation and improvement of cutting safety
}

\author{
Yao Yan · Jian Xu · Marian Wiercigroch
}

Received: date / Accepted: date

\begin{abstract}
This paper estimates cutting safety by using basin stability, and introduces state-dependent intermittent controls to improve it. Due to inherent nonlinearity and non-smoothness in tool-workpiece interactive forces, metal cutting processes often exhibit subcritical instability when they lose linear stability, inducing co-existence of large amplitude vibration, also called regenerative chatter, with stable stationary cutting. This phenomenon makes a considerable part of linearly stable region unsafe, where pure parameter selection is insufficient to maintain the cutting stability. To use the unsafe zones for higher material removal rate without incurring chatter, the possibility of chatter occurrence should be estimated as an index of cutting safety. This is achieved by basin stability estimation with delayed initial cutting states approximated by Fourier series and the coefficients generated based on Monte Carlo principle. It is found that the cutting safety in the "boundary layer" adjacent to linear stability boundaries is very severe, and a larger waviness height in workpiece surface can make the situation even
\end{abstract}

Y. Yan

School of Aeronautics and Astronautics, University of Electronic Science and Technology of China, Chengdu 611731, China

E-mail: y.yan@uestc.edu.cn

\section{J. $\mathrm{Xu}(\square)$}

Institute of AI and Robotics, Fudan University, 220 Handan Road, Shanghai 200433, China

Tel.: +86 13611872360

E-mail: jian_xu@fudan.edu.cn; xujian@tongji.edu.cn

M. Wiercigroch

Centre for Applied Dynamics Research, School of Engineering, Fraser Noble Building, King' s College, University of Aberdeen, Aberdeen, AB24 3UE Scotland, UK

E-mail: m.wiercigroch@abdn.ac.uk severer. To improve the safety by decreasing the possibility of chatter occurrence, state-dependent intermittent control with various perturbation strategies, i.e. linear and nonlinear velocity feedbacks and spindle speed variation (SSV), are proposed. With respect to the increase of control threshold or perturbation strength, the chatter orbits are gradually destroyed so that the majority of the UZs becomes globally stabilised.

Keywords Time-delayed cutting dynamics · Multiple stability · Basin stability · Unsafe cutting · Statedependent intermittent control

\section{Introduction}

As secondary or finishing operations, machining parts manufactured by other processes, such as casting, forming, or shaping, is critical for high quality surface finish, tight tolerances and drilling long and small diameter holes [13]. These highly depend on machining stability and suppression of unwanted vibration [39]. The source of cutting instability has been classified into frictional, regenerative, mode-coupling and thermo-mechanical types [42], and the regenerative is the most common one widely existing in various metal cutting operations, such as turning [36], milling [21], drilling [43] and grinding processes [52]. The regenerative instability is induced by successive material removal in tool-workpiece interaction, where instantaneous chip thickness depends on both current and previous tool passes [1]. The involvement of previous tool displacements makes the governing equations of cutting dynamics to be delayed differential equations (DDEs) [53]. Linear analysis of these models yields the so-called "lobes" 
diagrams, based on which parameters are selected to avoid chatter [38].

In the past two decades, results of this linear analysis have been being challenged as the nonlinear tool-workpiece interactions often promote large-amplitude chatter to co-exist with linearly stable cutting processes [47]. This multiple stability is called unsafe cutting (UC) as the cutting operation here has a level of possibility to vibrate even it is supposed to be stable base on the "lobes" diagrams. Stépán [34] presumed that the global cutting stability can be guaranteed only when the parameters are selected $8-10 \%$ lower than the linear stability boundary. It was thereafter realized that the $\mathrm{UC}$ is induced by subcritical Hopf bifurcation on stability boundaries, which bends unstable branches of periodic vibration towards the linearly stable zone until non-smoothness folds them back to yield large-amplitude chatter [22]. Based on this hypothesis, the unsafe zones (UZs) where chatter co-exists with stable stationary cutting can be estimated by tracking the unstable periodic solution and checking the non-smoothness [6]. Practically, the UZs are abandoned to avoid chatter, in spite of the fact that cutting efficiency is sacrificed.

When the UZs are used for higher cutting efficiency, initial conditions should be selected inside the basin of attraction of the steady cutting process to avoid chatter [44]. However, conventional basin of attraction is not easily applicable to cutting multiple stability as the DDEs governing cutting dynamics have infinite many dimensions [7]. This is a general problem with all DDEs, which requires a reasonable adjustment of the conventional basin of attraction. A simple example was proposed by Shang and $\mathrm{Xu}$ [31] and Shang [30], who hypothesised that there is no control signal before the time instant $t=0$ for systems with delays only in their feedbacks. Thus the conventional basin of attraction is applicable to this kind of systems. Based on this argument, they studied safe basin erosion of systems with delayed position, velocity and state feedbacks. In a similar manner, Höhne [8] artificially switched off delayed feedback for one period at the very beginning of a limit cycle oscillation, shrinking the infinite-dimensional space to be 2-dimensional for the selection of initial conditions. Alternatively, Ji [12] assumed constant initial conditions instead of time-varying functions to simplify the delayed states of a mass-damper-spring system with delayed feedbacks for the analysis of coexisting small- and large-amplitude vibrations. This method can be classified into the approximation of delayed initial functions by using polynomials. A systematic description of this concept was given by
Wang et al. [40] and Zheng and Sun [54], who employed the coefficients of polynomials to represent the functional space of delayed initial states.

Beside the polynomials' approach, $\mathrm{Hu}$ [9] argued that approximating delayed initial functions by Fourier series can be an alternative approach, and this would be especially useful for systems with oscillatory properties. Leng et al. [14] extended this idea by using three different orthogonal bases to randomly generate a large number of initial conditions to estimate the basin stabilities of two dynamical systems, which discovered that a selection of different orthogonal bases does not make too much difference. Then the approximation of delayed initial states by orthogonal sinusoid basis was employed by Yan et al. [51] to study the unsafe cutting, finding a narrow "boundary layer" in the UZs with large possibilities of chatter occurrence. It was also found that the cutting is prone to vibrate when the space frequency of workpiece surface is close to primary chatter frequency or its harmonics. However, this approach to some extent is over simplified as it uses only individual sinusoid function to approximate the initial functions. A better approximation requires combinations of the basis functions.

With such approximation, the basin of attraction or basin stability can be computed as an index of cutting safety in the UZs, based on which one can verify the effects of various control methods in improving the cutting safety. A simple and efficient method for higher cutting safety is continuous active or passive controls, such as linear and nonlinear feedbacks [25,37], spindle speed variations $[19,46]$, vibration absorbers [6,33], etc. These conventional methods changes dynamic properties of cutting processes, continuously requiring energy input for chatter suppression. Instead of this, an alternative method is to use the intrinsic dynamic properties of the cutting, which only requires a small energy expense to drive the chatter to the steady cutting. Yan et al. [51] have undertaken a very preliminary work in this area by introducing an impact to prevent large amplitude chatter and drive it towards the steady cutting. They estimated an impact strength and understood that it must be opposite to the tool's motion when the tool accelerates from zero velocity towards its maximum. This method is very efficient, but it may be hard to practically implement as it requires a large actuating force during a very short period of time.

A simple improvement of this is to divide one strong impact into "several small impacts" to accurately perturb and destroy the chatter orbit. This 
idea is a kind of state-dependent intermittent control, where the control is activated only when system state enters a predefined region and the intrinsic dynamic behaviour of the original system is preserved outside this region $[41,29]$. This idea was successfully adopted by Yadav et al. [45] to directionally switch the dynamics of a 3-dimensional system with 2 chaotic attractors. When the intermittent control law is designed properly, it is possible to directionally switch attractors by a single control action within a very short duration. A good example of this is the impulsive control used by Liu et al. [17]. Up to now, the effectiveness of controlling multiple stability in delayed systems by state-dependent intermittent control has not been discussed yet, and we are going to use it to improve the cutting safety discussed above.

To estimate the cutting safety and then to improve it, this paper is organised as follows. A brief introduction of the metal cutting dynamics and unsafe cutting is given in Section 2. Then Section 3 discusses the approximation of delayed initial functions and the evaluation of cutting safety. After that, three state-dependent intermittent control strategies are proposed and compared in Section 4. Finally, conclusions are drawn in Section 5.

\section{Unsafe cutting}

Cutting multiple stability has already been observed in various machining processes, such as the turning [23], milling [4], grinding [52], drilling [16], etc. This is normally induced by subcritical Hopf bifurcation born on linear stability boundaries, which generates an unstable branch of periodic orbit bending towards the linearly stable region [11]. This branch is then folded back by non-smoothness in the tool-workpiece interaction, loss of contact, to yield stable large-amplitude chatter co-existing with the steady cutting [50]. Due to the multiple stability, a stable cutting process cannot be guaranteed purely by parameter selection based on linear analysis, but requires proper initial conditions as well [35]. Therefore this operation is deemed as unsafe cutting (UC), and the region with multiple stability is called unsafe zones (UZs) [22,51].

The mechanism of UC can be revealed by bifurcation analysis with a very simple but widely accepted 1-dimensional turning model. Other cutting processes can be regarded as an extension of this as they all share the same intrinsic dynamic property, the delayed regenerative effect with non-smoothness in the cutting depth $[26,18,49,24]$.

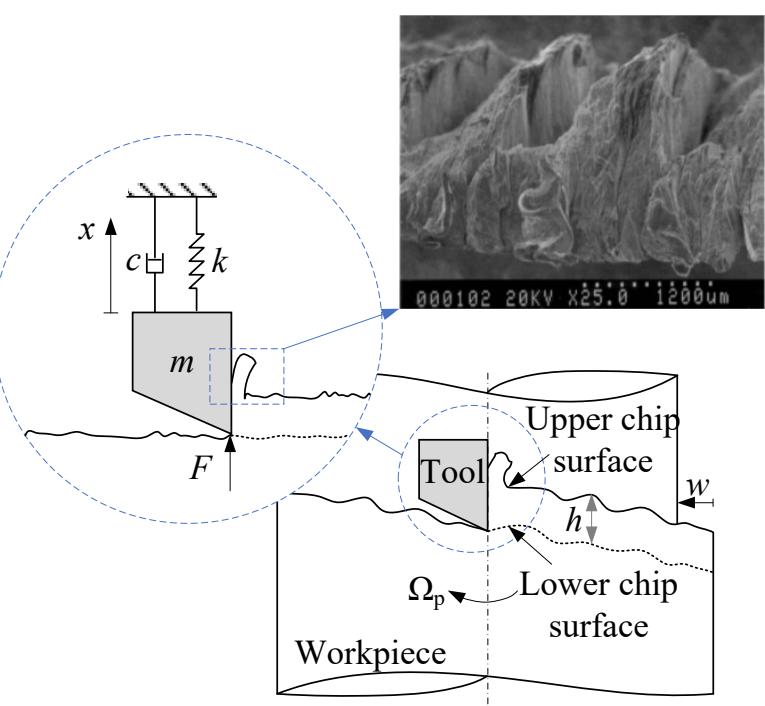

Fig. 1 Cutting dynamics with regenerative tool-workpiece interactive force generated in the chip formation process, where the scanning electron microscopy (SEM) image of the chip is adopted from Ref. [42]

\subsection{Simple regenerative cutting model}

Firstly, let us revisit the simplest classic dynamic model of regenerative cutting dynamics shown in Fig. 1 [5]. The workpiece rotates towards the cutting tool with an angular speed of $\Omega_{\mathrm{p}}\left[\mathrm{rad} \mathrm{s}^{-1}\right]$ while the tool moves downwards with a feeding speed of $h_{0}[\mathrm{~m}]$ per workpiece revolution. With equivalent mass of $m$ [kg], damping coefficient of $c\left[\mathrm{~N} \mathrm{~s} \mathrm{~m}^{-1}\right]$ and stiffness of $k\left[\mathrm{~N} \mathrm{~m}^{-1}\right]$, the tool has its vertical displacement governed by

$\ddot{x}(T)+2 \xi \omega_{\mathrm{n}} \dot{x}(T)+\omega_{\mathrm{n}}^{2} x(T)=\frac{F(T)}{m}$,

where $\omega_{\mathrm{n}}=\sqrt{\frac{k}{m}}\left[\mathrm{~s}^{-1}\right]$ and $\xi=\frac{c}{2 m \omega_{\mathrm{n}}}$ are the natural frequency and damping ratio of the tool, respectively. The tool-workpiece interactive cutting force, $F(T)[\mathrm{N}]$, is a nonlinear function of cutting depth $[32,22]$ :

$F(T)=w\left(\rho_{1} h(T)+\rho_{2} h(T)^{2}+\rho_{3} h(T)^{3}\right)$,

where $h(T)[\mathrm{m}]$ and $w[\mathrm{~m}]$ are instantaneous cutting depth (chip thickness) and width, and $\rho_{1}\left[\mathrm{~N} \mathrm{~m}^{-2}\right], \rho_{2}$ $\left[\mathrm{N} \mathrm{m}^{-3}\right]$ and $\rho_{3}\left[\mathrm{~N} \mathrm{~m}^{-4}\right]$ are coefficients [32]. Based on the regenerative theory shown in Fig. 1, the chip has its upper surface generated by previous tool pass, so that its thickness is represented by [23]

$h(T)=h_{0}+x\left(T-T_{\mathrm{p}}\right)-x(T)$,

where $T_{\mathrm{p}}=\frac{2 \pi}{\Omega_{\mathrm{p}}}[\mathrm{s}]$ is the rotation period of the workpiece. 
By introducing the following dimensionless variables and parameters [51]

$t=\omega_{\mathrm{n}} T, \quad d=\frac{h}{h_{0}}, \quad \tau=\omega_{\mathrm{n}} T_{\mathrm{p}}, \quad \Omega=\frac{\Omega_{\mathrm{p}}}{\omega_{\mathrm{n}}}$,

$y_{1}=\frac{x}{h_{0}}-\frac{w}{m \omega_{\mathrm{n}}^{2}}\left(\rho_{1}+\rho_{2} h_{0}+\rho_{3} h_{0}^{2}\right), \quad y_{2}=\frac{\mathrm{d} y_{1}}{\mathrm{~d} \tau}$

$\eta_{1}=\frac{w}{m \omega_{\mathrm{n}}^{2}}\left(\rho_{1}+2 \rho_{2} h_{0}+3 \rho_{3} h_{0}^{2}\right)$,

$\eta_{2}=\frac{w}{m \omega_{\mathrm{n}}^{2}}\left(\rho_{2} h_{0}+3 \rho_{3} h_{0}^{2}\right), \quad \eta_{3}=\frac{w}{m \omega_{\mathrm{n}}^{2}} \rho_{3} h_{0}^{2}$,

the regenerative model, Eq. (1), is simplified into

$\mathbf{y}^{\prime}(t)=\mathbf{A y}(t)+\mathbf{D} \mathbf{y}(t-\tau)+\mathbf{N}$,

where the prime represents the derivative with respect to the dimensionless time, $t$, and the matrices are

$\mathbf{y}(t)=\left(\begin{array}{l}y_{1} \\ y_{2}\end{array}\right), \quad \mathbf{A}=\left(\begin{array}{cc}0 & 1 \\ -1-\eta_{1}-2 \xi\end{array}\right), \quad \mathbf{D}=\left(\begin{array}{cc}0 & 0 \\ \eta_{1} & 0\end{array}\right)$,

$\mathbf{N}=\left(\begin{array}{c}0 \\ \eta_{2}\left(y_{1}(t-\tau)-y_{1}(t)\right)^{2}+\eta_{3}\left(y_{1}(t-\tau)-y_{1}(t)\right)^{3}\end{array}\right)$.

In Eq. (4), $\tau$ and $\Omega$ are the dimensionless rotational period and speed of the workpiece, and $\eta_{1}, \eta_{2}$ and $\eta_{3}$ are dimensionless coefficients of the cutting force. The dimensionless time, chip thickness, tool displacement and velocity are represented by $t, d, y_{1}$ and $y_{2}$, respectively. When the cutting is static without tool vibration, one has $y_{1}=0$ and $d=1$ corresponding to the equilibrium of Eq. (5). This static state is expected to be stable for stationary cutting, which will be discussed in the following analysis via eigenvalue analysis.

\subsection{Unsafe cutting}

By using eigenvalue analysis (See more details in Ref. [5]), one can find the following parametric functions of the critical "lobes" curves

$$
\begin{aligned}
& \eta_{1 \mathrm{c}}(\omega)=\frac{\left(\omega^{2}-1\right)^{2}+4 \xi^{2} \omega^{2}}{2\left(\omega^{2}-1\right)} \\
& \tau_{\mathrm{c}}(\omega)=\frac{2}{\omega}\left(i \pi-\arctan \left(\frac{\omega^{2}-1}{2 \xi \omega}\right)\right), \quad i=1,2,3, \cdots,
\end{aligned}
$$

where $\eta_{1 \mathrm{c}}$ and $\tau_{\mathrm{c}}$ represent the critical values of $\eta_{1}$ and $\tau$ on stability boundaries, and $\omega \in(1,+\infty)$ is the chatter frequency. Letting $\eta_{\min }=2 \xi(1+\xi)$ be the minimum of $\eta_{1 c}$ and using $\xi=0.01, \rho_{1}=6109.6, \rho_{2}=-54141.6$ and $\rho_{3}=203769[5,32]$, one obtains the typical "lobes" curves (solid) shown in Fig. 2 to separate the linearly stable (below) and unstable (above) regions.

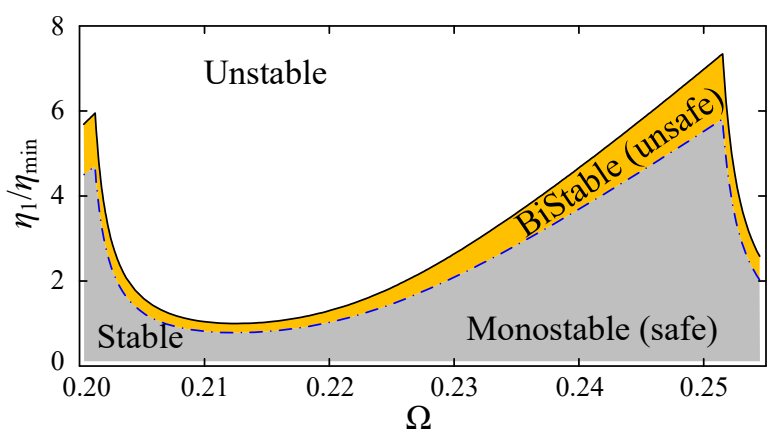

Fig. 2 'Lobes' diagram for the cutting stability, where the solid lines separates the linearly stable and unstable regions, while the stable one is further divided by the dot-dashed lines into unsafe (bistable) and safe (monostable) zones.

By applying bifurcation analysis on the solid curves and tracking the unstable periodic solutions, one can further obtains the boundaries (dot-dashed) of the UZs (yellow) in Fig. $2[23,52]$. To determine the long-term cutting dynamics in the UZs, the initial conditions should be considered as well besides the parameters. However, due to the infinitely-many dimensions introduced by the inherent delay in Eq. (5), one cannot study the cutting multistability by conventional basin of attraction [28]. Thus a reasonable approximation of the delayed initial states is required to calculate basin stability and evaluate cutting safety. To this end, we will revisit the method used in Ref. [51] and then extend it with a random combination of basis functions.

\section{Estimation of cutting safety}

The cutting process in Fig. 1 is replotted in the left part of Fig. 3(a), where its outmost layer is to be removed by successive tool passes. This layer is unfolded and mapped onto the $\theta-p$ coordinate in the right of Fig. 3(a), showing the tool passes on the workpiece surface. There are three kinds of tool passes in Fig. 3(a), namely the unbent $\left(p_{\mathrm{u}}\right)$, static $\left(p_{\mathrm{s}}\right)$ and dynamic $\left(p_{\mathrm{d}}\right)$ ones, respectively corresponding to the unbent (dashed), statically bent (solid) and dynamical bent (thick solid) tools displayed in Fig. 3(b).

Before the tool contacts with the workpiece, it is unbent $(x \equiv 0)$, so Eq. (4) yields

$$
\begin{aligned}
y_{1} \equiv y_{10} & =-\frac{w}{m \omega_{\mathrm{n}}^{2}}\left(\rho_{1}+\rho_{2} h_{0}+\rho_{3} h_{0}^{3}\right) \\
& =-\left(\eta_{1}-\eta_{2}+\eta_{3}\right),
\end{aligned}
$$

for the unbent tool pass, $p_{\mathrm{u}}$. When the cutting begins, the tool pass is expected to be static $\left(p_{\mathrm{s}}\right)$ without any chatter, which corresponds with $y_{1} \equiv 0$. However, the 


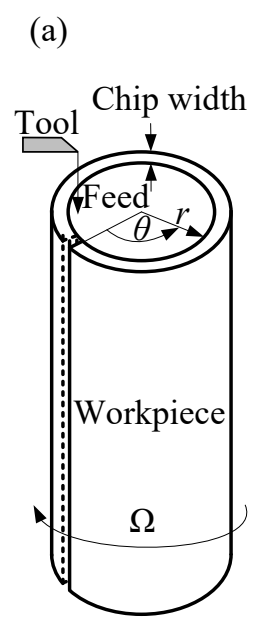

(b)

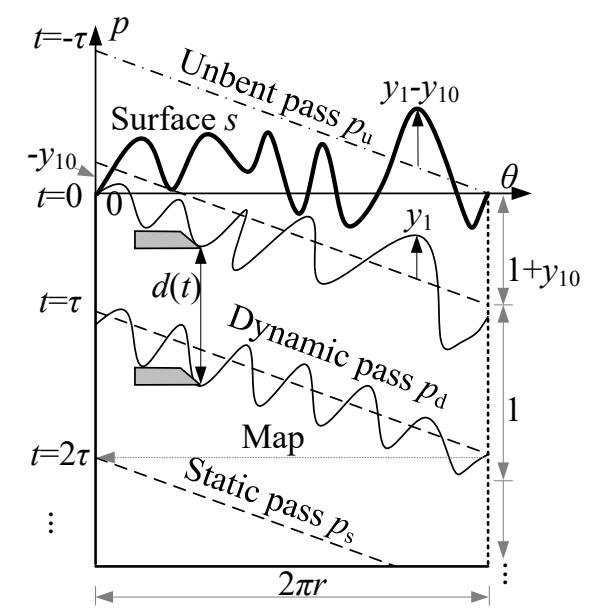

(c)
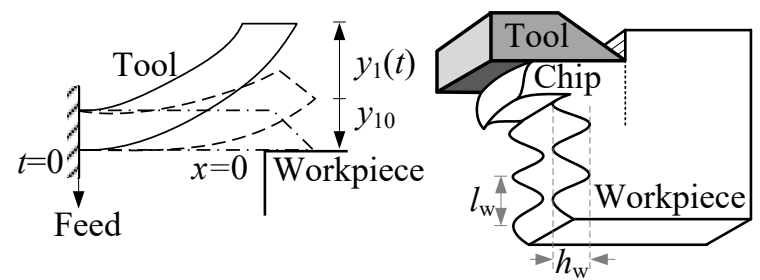

Fig. 3 (a) The outmost layer of the workpiece to be cut is mapped onto $\theta-p$ plane, where the thick curve represents the workpiece surface, $s$. The dot-dashed line is unbent tool pass, $p_{\mathrm{u}}$, before it engages with the workpiece, the dash lines are static tool passes, $p_{\mathrm{s}}$, and the solid curves are the dynamics passes , $p_{\mathrm{d}}$, involving tool vibration. (b) Unbent (dot-dashed), statically bent (dashed) and dynamically bent (solid) tools. (b) Initial workpiece surface with waviness height, $h_{\mathrm{w}}$, and wavelength, $l_{\mathrm{w}}$.

tool is often perturbed to follow the dynamic tool pass oscillating around the static one, with the difference

$y_{1}=y_{1}-0=p_{\mathrm{d}}-p_{\mathrm{s}}$,

as the dimensionless tool displacement governed by Eq. (5). Equivalently, the initial wavy surface of the workpiece, $s$, can be regarded as an imaginary dynamic tool pass before the cutting starts, which oscillates around the unbent pass, $p_{\mathrm{u}}$, with the difference

$y_{1}-y_{10}=s-p_{\mathrm{u}}$.

From Fig. 3(a), it is seen that the distance between the unbent pass and the first static pass, which is $1+y_{10}$, is smaller than the distance between successive static passes which is 1 . This is due to the static deformation of the tool after it engages with the workpiece, which gradually bends the tool from $x=0$ towards $y=0$ during the tool cutting into the workpiece.
As depicted in Fig. 3(a), $s$ and $p_{\mathrm{u}}$ are functions of $\theta$ as follows

$s=s(\theta)$,

$p_{\mathrm{u}}=p_{\mathrm{u}}(\theta)=1-\frac{\theta}{2 \pi}, \quad \theta \in[0,2 \pi]$.

When perturbations, such as chip breakage, external noise and etc., are ignored, $s(\theta)$ can be treated as a periodic function as the workpiece is cylindrical, which has

$s(\theta+2 \pi)=s(\theta)$.

If external perturbations during the tool cutting into the workpiece is considered, one can add an extra random function to mimic its effect. With respect to the workpiece rotation, the angle, $\theta$, before tool-workpiece engagement $(t=0)$ can be regarded as a function of time as follows

$\theta(t)=2 \pi+\frac{t}{\tau} 2 \pi, \quad t \in[-\tau, 0]$.

Next, substituting Eqs (10), (11) and (12) into Eq. (9) and using $\Omega \tau=2 \pi$ yield the function for the delayed initial states as follows

$$
\begin{aligned}
y_{1}(t) & =s(\theta(t))-p_{\mathrm{u}}(\theta(t))+y_{10} \\
& =s\left(2 \pi+\frac{t}{\tau} 2 \pi\right)+\frac{t}{\tau}+y_{10} \\
& =s(\Omega t)+\frac{\Omega}{2 \pi} t+y_{10}, \quad t \in[-\tau, 0] .
\end{aligned}
$$

At the instant of $t=0$, this equation yields $y_{1}(0)=$ $s(0)+y_{10}$, while it is also known that the tool is unbent $\left(y_{1}(0)=y_{10}\right)$ when the cutting is about to begin. This yields a boundary condition of $s(\theta)$ as follows

$s(0)=0$.

\subsection{Approximation of initial functions}

In Eq. (13), only the term, $s(\Omega t)$, is undefined for the selection of the initial function, $y_{1}(t)(t \in[-\tau, 0])$. It is known from Eqs (11) and (14) that $s(\Omega t)$ is a periodic function with $s(2 \pi)=s(0)=0$. These properties indicate that a series of sinusoid functions can be used to approximate $s(\Omega t)$. In addition, given the hypothesis [9] that Fourier basis is a good choice to approximate initial functions of delayed systems with oscillatory dynamic properties, the following approximation is used

$$
\begin{aligned}
s(\Omega t) \approx & \sum_{i=1}^{N} a_{i} \sin (i \Omega t+i \phi)-a_{i} \sin (i \phi) \\
& +\sum_{i=1}^{N} b_{i} \cos (i \Omega t+i \phi)-b_{i} \cos (i \phi),
\end{aligned}
$$


where $a_{i}, b_{i}$ and $\phi$ are undetermined parameters. The selection of $a_{i}$ and $b_{i}$ determines the waviness height and wavelength, $h_{\mathrm{w}}$ and $l_{\mathrm{w}}$, of the workpiece surface illustrated in Fig. 3(c), while $\phi$ depends on the geometry of tool-workpiece engagement when the cutting begins [51]. Beside the Fourier basis used here, it should be remarked that other orthogonal bases, such as Chebyshev and Legendre polynomials, can be used as well. Since the selection of basis functions has already been discussed by Leng et al. [14], who found no significant difference in their investigation of time delayed systems, the following analysis will use Eq. (15) to approximate the functional initial conditions and other basis functions will be employed in our future works.

\subsection{Basin of attraction approach}

Given Eq. (15), the basin of attraction of the UC can be defined in a $(2 N+1)$-dimensional parameter space, $a_{i}-b_{i}-\phi(i=1,2, \cdots, N)$, which requires excessive amount of simulations. Moreover, visualization of this high-dimensional results would be very difficult if not impossible [3]. Alternatively, a simplified version of Eq. (15) with $b_{i}=\phi=0$,

$s(\Omega t) \approx \sum_{i=1}^{N} a_{i} \sin (i \Omega t)$,

has already been employed by Yan et al. [51] to approximate the initial functions. In addition, to reveal the effect of individual harmonic on the possibility of chatter occurrence, they [51] did not combine the basis functions but successively investigated each harmonic.

In this simplified case, it is possible to construct an equivalent basin of attraction shown in Figs 4(c), (d) and (e), where the initial functions selected in the black regions go towards chatter but the white regions lead to stable cutting. It is seen that the black regions are accumulated around the primary chatter frequency and its sub- and super-harmonics, so that the basin stability for each harmonic, $S_{i}$, reaches its peaks around $\frac{\omega}{2}, \omega$ and $2 \omega$. Letting $A_{\mathrm{s}}$ and $A_{\mathrm{c}}$ represent the areas of the white and black regions, one can define

$S_{\mathrm{Bc}}=\frac{A_{\mathrm{c}}}{A_{\mathrm{c}}+A_{\mathrm{s}}} \times 100 \%$ and $S_{\mathrm{Bs}}=100 \%-S_{\mathrm{Bc}}$,

to represent the basin stabilities of chatter and steady cutting, respectively. As seen in Figs 4(a) and (b), the basin stability of chatter, $S_{\mathrm{Bc}}$, monotonously increases with respect to the increase of $\eta_{1} / \eta_{\min }$ towards the linear stability boundary, and the trend of growth is
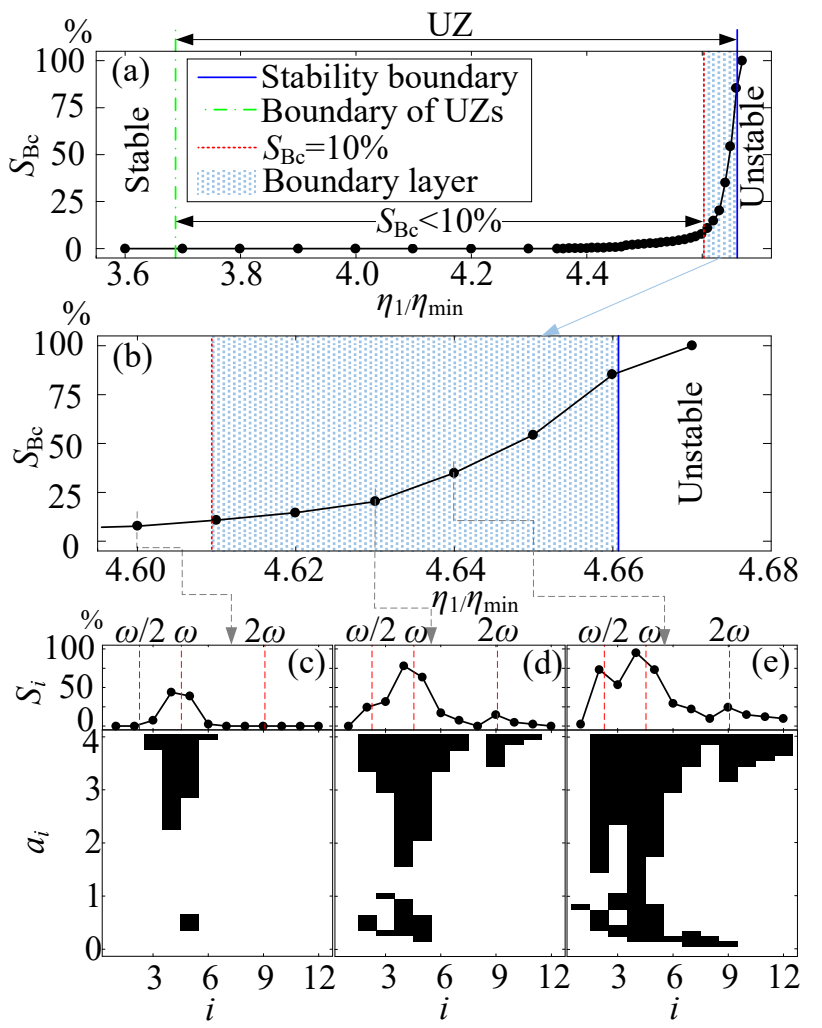

Fig. 4 (a) Basin stability of the chatter, $S_{\mathrm{Bc}}$, for $\Omega=0.24$ is plotted as a function of $\eta_{1} / \eta_{\min }$, with the region adjacent to the stability boundary enlarged in Panel (b) and the basins of attraction for $\eta_{1} / \eta_{\min }=4.6,4.63$ and 4.64 displayed in Panels (c), (d) and (e) [51].

accelerated in the "boundary layer" adjacent to the stability boundary, indicating a severe cutting safety in this zone.

\subsection{Basin stability estimation}

Although the above simplified approach can reveal the effects of individual harmonics on the cutting safety, Eq. (16) to some extent is not precise enough to approximate real workpiece surface described by Eq. (15), not to mention the absence of the combination of basis functions in the construction of basin of attraction in Fig. 4. Thus the following discussion will be based on Eq. (15), with random combinations of the basis functions. However, only basin stability will be used next as a high dimensional basin of attraction is difficult to visualize [3].

The basin stability is estimated based on Monte Carlo principle [2], which considers $a_{i}, b_{i}$ and $\phi$ as random numbers uniformed distributed in predefined intervals. For a limited waviness height, $h_{\mathrm{w}}$, we can use an amplitude bound, $\alpha$, to confine the selection of 


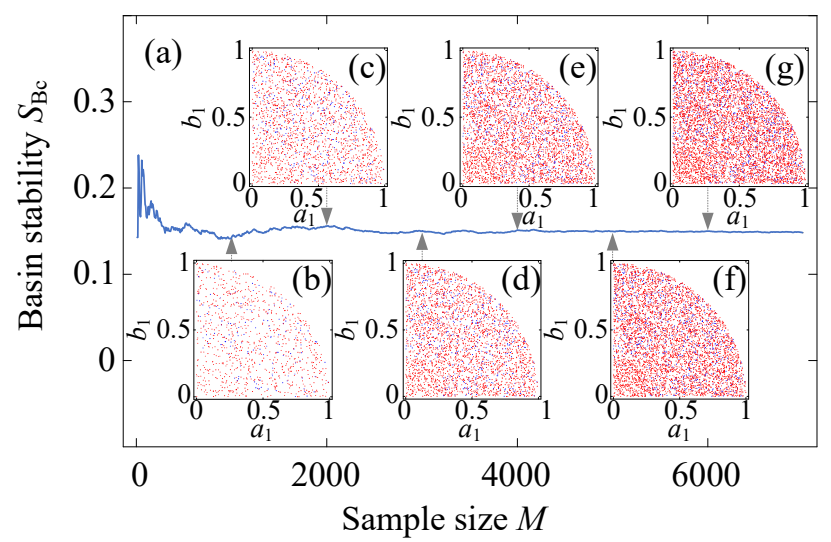

Fig. 5 Influence of the sample size, $M$, on the convergence of the basin stability, $S_{\mathrm{Bc}}$, for $\Omega=0.24, \eta_{1} / \eta_{\min }=4.64$, $N=1$ and $\alpha=1$. The randomly generated coefficients, $\left\{\left(a_{1}^{(j)}, b_{1}^{(j)}\right)\right\}_{j=1}^{M}$, for $M=1000,2000,3000,4000,5000$ and 6000 are displayed in Panels (b-g), where the red and blue dots go to steady cutting and chatter, respectively.

$a_{i}$ and $b_{i}$ as [14]

$\sum_{i=N}^{N} a_{i}^{2}+b_{i}^{2} \leq \alpha^{2}$.

In addition, $\phi$ can be restricted as follows without loss of generality

$\phi \in[0,2 \pi)$.

Letting $M$ be the sample size, the Monte Carlo principle generates $M$ groups of parameters, $\left\{\left(a_{1}^{(j)}, \cdots, a_{N}^{(j)}, b_{1}^{(j)}, \cdots, b_{N}^{(j)}, \phi^{(j)}\right)\right\}_{j=1}^{M}$, corresponding with $M$ initial functions. If $M_{\mathrm{c}}$ out of $M$ groups lead to the chatter in the UZs, the basin stabilities of the chatter and steady cutting are respectively estimated as

$S_{\mathrm{Bc}}=\frac{M_{\mathrm{c}}}{M} \times 100 \%$ and $\quad S_{\mathrm{Bs}}=100 \%-S_{\mathrm{Bc}}$.

A simple example of this estimation is illustrated in Fig. 5 , where $\Omega=0.24, \eta_{1} / \eta_{\min }=4.64, N=1$ and $\alpha=1$ are used. In Panels (b-g), the randomly generated $\left\{\left(a_{1}^{(j)}, b_{1}^{(j)}\right)\right\}_{j=1}^{M}$ are displayed with the blue and red points leading to chatter and stable cutting, respectively. While the points distribution in Figs 5(b-g) becomes denser and denser with respect to the increase of the sample size, the basin stability, $S_{\mathrm{Bc}}$, gradually convergent for $M \geq 2000$. The same trend is also observed in Fig. 6(a), where the basin stabilities always convergent for $M \geq 2000$, no matter how many basis functions are used.

Next we consider the influence of the number of basis functions, $N$, on the convergence of $S_{\mathrm{Bc}}$. It is seen in Figs 4(c), (d) and (e) that higher order

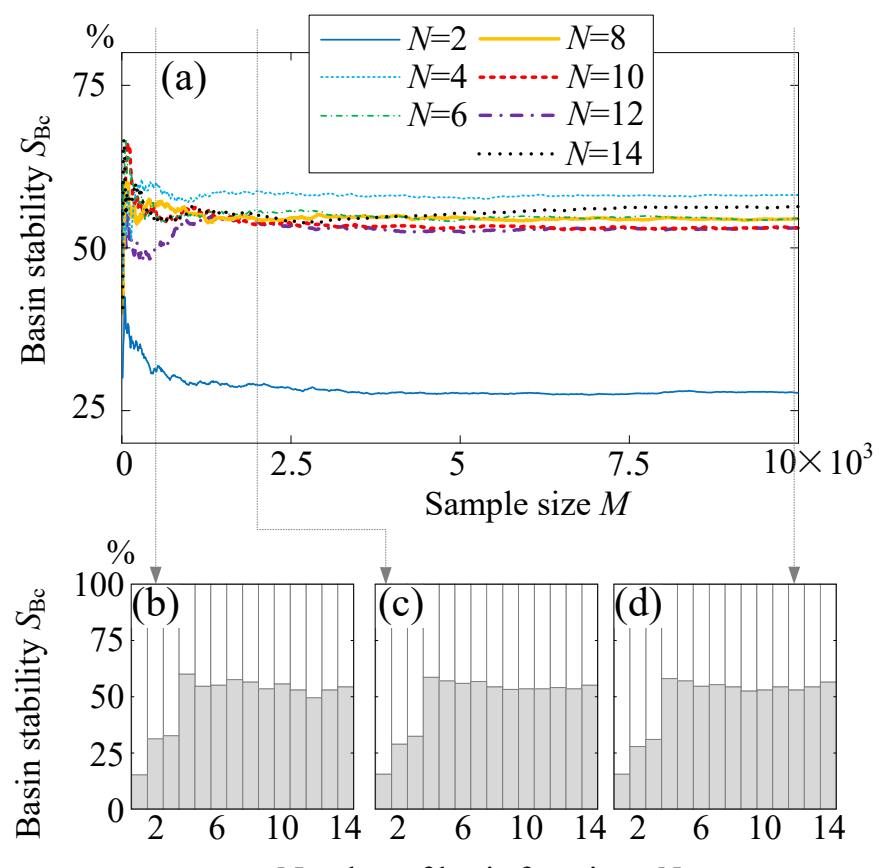

Number of basis functions $N$

Fig. 6 (a) Basin stabilities of chatter, $S_{\mathrm{Bc}}$, for $\Omega=0.24$, $\eta_{1} / \eta_{\min }=4.64$ and $\alpha=1$, with $M$ and $N$ undetermined. Bar charts for (b) $M=1000$, (c) 2000 and (d) 10000 are added to illustrate the influence of $N$.

harmonics hardly make any contribution to the chatter occurrence in the UZs [52]. Thus the basin stabilities in Figs 6(b), (c) and (d) gradually convergent with respect to the increase of the number of basis functions for $N>8$. Simply put, there is no significant difference in the estimation of $S_{\mathrm{Bc}}$ with a different sample size or number of basis functions provided that $M$ and $N$ are large enough.

Finally, the influence of the amplitude bound, $\alpha$, is studied in Fig. $7(\mathrm{a})$, where $\Omega=0.24, \eta_{1} / \eta_{\min }=4.64$ and $N=10$ are used. It is seen that $S_{\mathrm{Bc}}$ always convergent for $M \geq 2000$ no matter what the value of $\alpha$ is selected in $[0.5,4]$, which confirms our above argument. In addition, Fig. 7(b) illustrates that a possibility of chatter occurrence grows monotonously with respect to the increase of the amplitude bound, which is very fast for $\alpha<2$ but gradually level off to $100 \%$ thereafter. This warns us that the waviness height has a very significant influence on the cutting safety, and a smoother workpiece surface is helpful for chatter avoidance in the UZs. In practice, it is reasonable to presume that the waviness would not be larger than chatter marks left by a failure cutting operation, so the following analysis will use $\alpha=1$ as the amplitude bound of the surface waviness.

Based on the above discussion, the following analysis will employ $N=10, M=2000$ and $\alpha=1$ to 

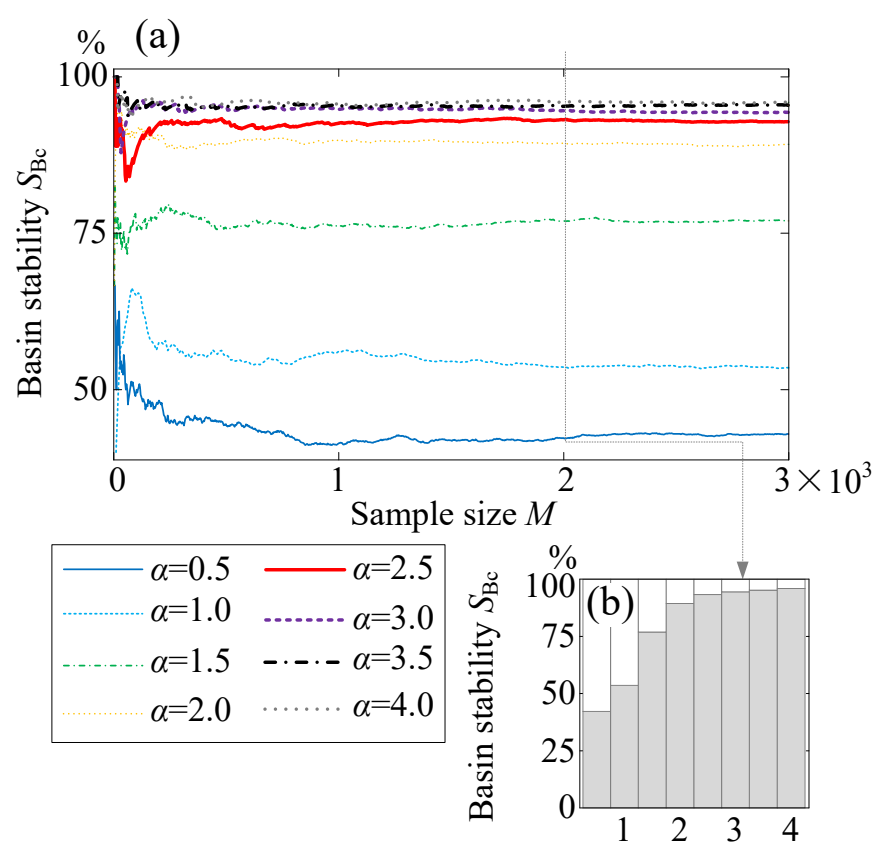

Amplitude bound $\alpha$

Fig. 7 (a) Basin stabilities of chatter, $S_{\mathrm{Bc}}$, for $\Omega=0.24$, $\eta_{1} / \eta_{\min }=4.64, N=10$ and various values of $\alpha$ are plotted as functions of $M$, with the (b) bar chart for $M=2000$ added to display the influence of $\alpha$.
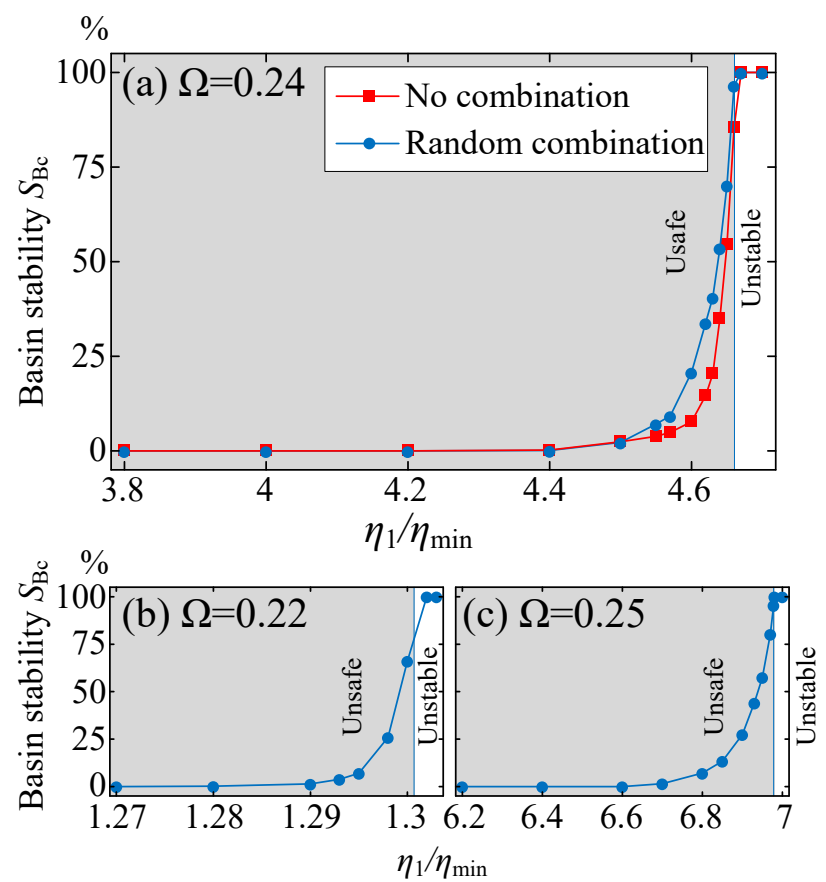

Fig. 8 (a) Comparison of the basin stabilities for $\Omega=0.24$ obtained without and with random combinations of basis functions for initial function approximation. Evolutions of $S_{\mathrm{Bc}}$ with respect to the increase of $\eta_{1} / \eta_{\min }$ for (b) $\Omega=0.22$ and (c) 0.25 are displayed as well. estimate the basin stability, i.e. cutting safety. This estimation is firstly compared with the result obtained by using Eq. (16) without combinations of the basis functions. Figure 8(a) shows a slight difference between the estimations obtained by the simplified approximation [51] and based on the Monte Carlo principle. Nonetheless, the growing trend of $S_{\mathrm{Bc}}$ with respect to the increase of $\eta_{1} / \eta_{\min }$ is the same, which has a very low possibility of the chatter occurrence in the majority of the UZs but the cutting safety significantly decrease in the "boundary layer" adjacent to the stability boundary. Similar results for $\Omega=0.22$ and 0.25 are displayed in Figs 8(b) and (c), which show the same trend along the stability boundary. This phenomenon is in accordance with the conclusions drawn in Ref. [52].

\section{Improving cutting safety by state-dependent intermittent perturbation}

Next the decrease of the basin stability of chatter, $S_{\mathrm{Bc}}$, will be used as an index to monitor the improvement of cutting safety. In Ref. [52], a single impulsive force was employed to perturb the orbit of large-amplitude chatter to successfully quench it. However, this simple method could be very hard for application as it requires a single accurate and strong control force applied in a very short period. Thus a gentler control strategy is expected to continuously disturb the chatter orbit with a relatively small control force. In addition, the control should accurately disturb the chatter orbit only so that the intrinsic dynamic property of the cutting can be preserved. That is, the control power is unnecessary once the cutting dynamics is already on its trajectory towards the stationary cutting. Unlike continuous control laws, this kind of multistability control keeps absent even when a small-amplitude vibration around the target has been promoted by external noise, so that its energy requirement can be very small [15].

The above idea is actually a state-dependent intermittent control, where the control is active only in a predefined region in the state space [45]. Introduction of this control transforms the governing equation of the cutting dynamics, Eq. (5), into

$\mathbf{y}^{\prime}(t)=\mathbf{A y}(t)+\mathbf{D y}(t-\tau)+\mathbf{N}+\mathbf{U}$,

where $\mathbf{U}=(0, h(\mathbf{y}) u(t))^{\mathrm{T}}$ is the state-dependent intermittent control. Here $h(\mathbf{y})$ is a non-smooth state-dependent function given by [45]

$h(\mathbf{y})=\left\{\begin{array}{lll}1 & \text { if } & \mathbf{y} \in S^{2} \\ 0 & \text { if } & \mathbf{y} \notin S^{2}\end{array}\right.$ 

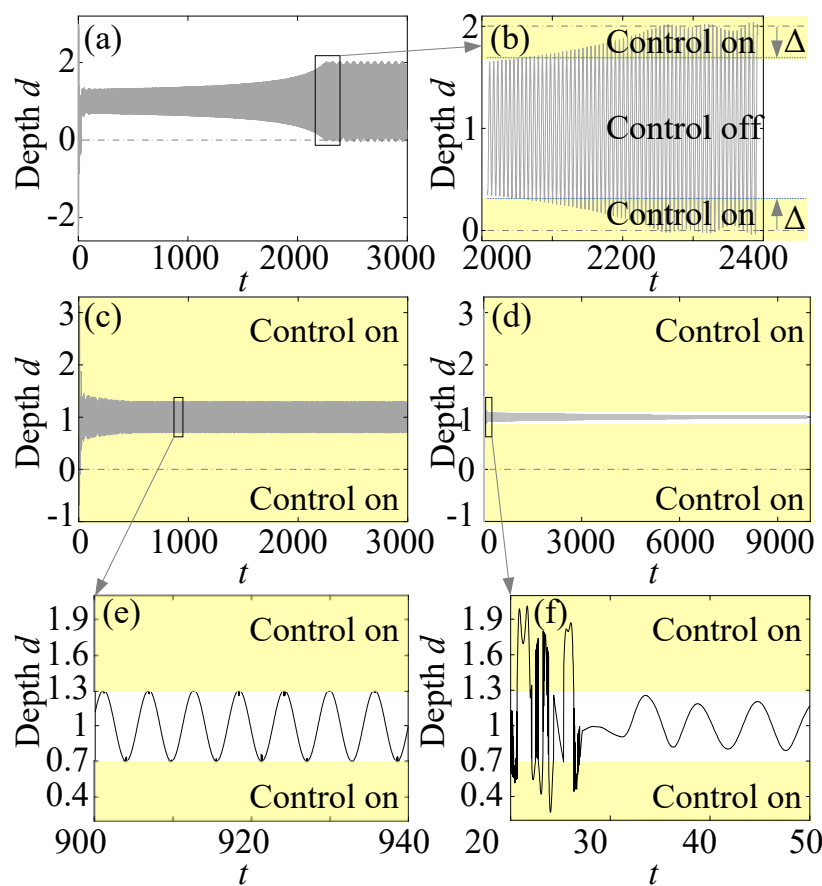

Fig. 9 (a) Time series of the regenerative chatter for $\Omega=0.24$ and $\eta_{1} / \eta_{\min }=4.64$, with the section for $t \in$ [2000,2400] enlarged in Panel (b) to illustrate the statedependent intermittent control. (c) A success and (b) a failure of chatter suppression by state-dependent intermittent control are displayed, with parts of the time series respectively enlarged in Panels (e) and (f).

where $S^{2}$ is the predefined subspace of the full state space $R^{2}$. Thus the control, $u(t)$, is active only for $\mathbf{y} \in$ $S^{2}$.

To improve the cutting safety in the UZs, the large amplitude chatter displayed in Fig. 9(a) is expected be suppressed to make the cutting dynamics monostable. Thus part of the chatter orbit should be included in a subspace $S^{2}$ for disturbing but the stationary cutting $(d \equiv 0)$ should be excluded for the absence of control force. A simple subspace of this kind is depicted as the yellow regions in Fig. 9(b) and described as follows

$$
\begin{aligned}
S^{2} & =\left\{\mathbf{y} \mid \operatorname{Abs}(d-1) \geq 1-\Delta, y_{2} \in R^{1}\right\} \\
& =\left\{\mathbf{y} \mid \operatorname{Abs}\left(y_{1}(t-\tau)-y_{1}(t)\right) \geq 1-\Delta, y_{2} \in R^{1}\right\}
\end{aligned}
$$

where $\operatorname{Abs}(a)$ takes the absolute value of $a$, and $\Delta \in$ $[0,1]$ is the threshold for the switch of control. For $\Delta=$ 1 , the control is always active (continuous), while $\Delta=0$ keeps the perturbation inactive until the tool loses its contact with the workpiece.

Once $\Delta$ is fixed, the most important factor influencing the cutting safety is the selection of the control law in $S^{2}$. To illustrate, Figs $9(\mathrm{c})$ and (d) select the same initial condition and threshold

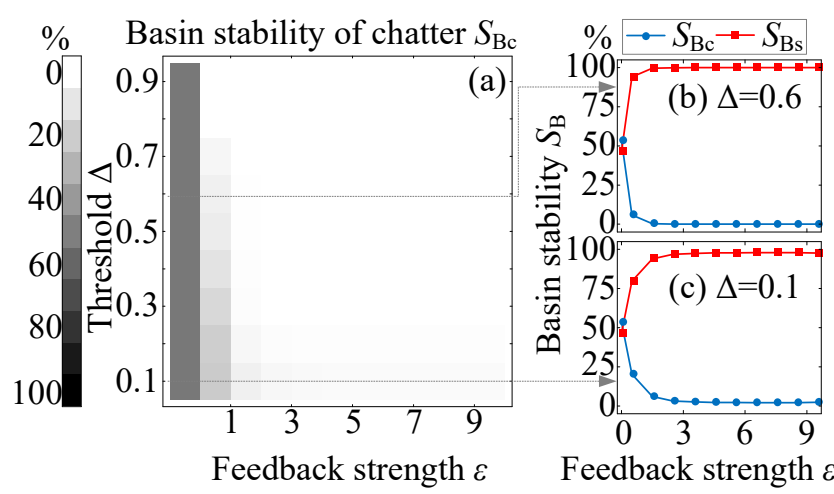

Fig. 10 (a) Effect of the state-dependent intermittent control with linear velocity feedback on the basin stability, where the chatter occurrence for different threshold and feedback strength is represented by the grayscale. Basin stabilities for $\Delta=0.6$ and 0.1 are plotted as functions of $\epsilon$ in Panels (b) and (c).

( $\Delta=0.7)$ but use different control laws, where the first control law fails but the second one successes in quenching the chatter. More details are given in the blown-up windows in Figs 9(e) and (f). The unsuccessful case in Fig. 9(e) forces the cutting depth, $d$, to oscillate between the switch surfaces. Although it reduces the chatter amplitude, it cannot completely eliminate the unwanted vibration. By contrast, the oscillation shown in Figs $9(\mathrm{~d})$ and (f) enters $S^{2}$ only before $t=30$ for several times, and it is gradually damped out thereafter without any control force. Next, the effects of three different control laws, including linear and nonlinear feedbacks and spindle speed variation (SSV) will be studied.

\subsection{Linear velocity feedback}

A simple method to perturb the chatter orbit is increasing the damping to dissipate more mechanical energy. Thus the first control law selected for the state-dependent intermittent perturbation is

$u(t)=-\epsilon y_{2}(t)$,

where $\epsilon$ is the strength of the negative linear velocity feedback. It is worth noting that Eq. (24) is only an idealized linear control, and time delays always exist in the feedback loop in practice. However, the feedback delay is neglected here to keep the discussion as simple as possible, but it will be considered in our future works.

To verify the effectiveness of this feedback, $\Delta \in[0.1,0.9]$ and $\epsilon \in[0,9.5]$ are used to improve the cutting safety for $\Omega=0.24$ and $\eta_{1} / \eta_{\min }=6.46$, which has $53.6 \%$ chance to vibrate when no control is applied. The basin stability of the chatter, $S_{\mathrm{Bc}}$, is 


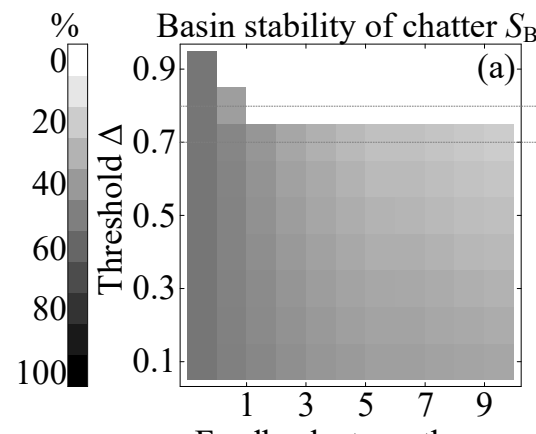

Feedback strength $\varepsilon$
$\% \rightarrow S_{\mathrm{Bc}}-S_{\mathrm{Bs}}$

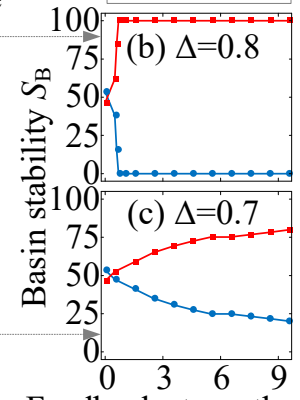

Feedback strength 8
Fig. 11 (a) Effect of the state-dependent intermittent control with cubic nonlinear velocity feedback on the basin stability, where the chatter occurrence for different threshold and feedback strength is represented by the grayscale. Basin stabilities for $\Delta=0.8$ and 0.7 are plotted as functions of $\epsilon$ in Panels (b) and (c).

illustrated by the greyscale in Fig. 10(a), where the greyest region is the left-most column for no control force $(\epsilon=0)$. For any specified threshold, $\Delta$, the greysacle uniformly decreases with respect to the increase of $\epsilon$, and this trend is especially evident when $\Delta$ is large. For a small threshold $(\Delta=0.1), S_{\mathrm{Bc}}$ in Fig. 10(c) decreases fast for $\epsilon<3$ but levels off to a small value thereafter. As $S_{\mathrm{Bc}}$ never reduces to zero even when it is very small, $\Delta=0.1$ cannot make the UC $100 \%$ safe. By contrast, $S_{\mathrm{Bc}}$ for a large threshold ( $\Delta=0.6)$ immediately becomes zero for $\epsilon>1.5$ in Fig. 10(b), indicating monostability for the safe cutting.

\subsection{Cubic nonlinear velocity feedback}

Beside the linear velocity feedback, cubic nonlinear damping can also be used to dissipate the chatter energy. A continuous cubic velocity feedback has already been employed by Pratt and Nayfeh [27] and Nayfeh and Nayfeh [25] to manipulate the bifurcation pattern in the cutting dynamics, where the subcritial instability was successfully transformed into supercritical when a large feedback strength was applied. This method was then verified by Yan et al. [48], who successfully suppressed the unwanted large-amplitude chatter in a regenerative grinding process. As an effective continuous control method for cutting safety improvement, its corresponding intermittent type,

$u(t)=-\epsilon y_{2}(t)^{3}$,

is to be tested next.

The effect of the state-dependent intermittent control with cubic nonlinear velocity feedback is displayed in Fig. 11. Unsurprisingly, its overall effectiveness in improving the cutting safety is less compared with Fig. 10 for the linear feedback as the nonlinear control force is usually smaller than the linear one when the vibration amplitude is small. However, a very attractive result shown in Fig. 11 points out an existence of a critical threshold ( $\Delta=0.8)$ for safe cutting. For a threshold below this critical value, e.g. $\Delta=0.7$, the increase of the feedback strength can indeed improve the cutting safety in Fig. 11(c), but it never yields $S_{\mathrm{Bc}}=0 \%$ for a $100 \%$ safe cutting process. By contrast, $\Delta=0.8$ in Fig. 11(b) only requires a very small feedback strength $(\epsilon \geq 0.7)$ to make the cutting $100 \%$ safe. This indicates that increasing the threshold, $\Delta$, for a larger active region is more important than enhancing the feedback strength, $\epsilon$. In practice, the measurement error of $y_{2}(t)$ can be enlarged by the cubic feedback, so its application should be performed with caution.

\subsection{Spindle speed variation}

Except the velocity feedbacks, another effective method for chatter suppression is spindle speed variation, which is a kind of open loop perturbation. The effect of continuous SSV has already been successfully verified in turning [10], milling [20], and grinding [46] dynamics analyses. Instead of a constant spindle speed, $\Omega$ is periodically altered to be

$\tilde{\Omega}(t)=\Omega\left(1+R V A \sin \left(\omega_{m} t\right)\right)$,

where $R V A$ is the ratio of speed varying amplitude to the normal spindle speed, and $\omega_{m}$ is the variation frequency. It known that the variation frequency is not as significance as $R A V$ for the effect of SSV provided it is larger than and is not resonant with the chatter frequency [46], so the following analysis will use $\omega_{m}=2$ and leave $R V A$ undetermined. However, it should be noted that $\omega_{m}=2$ might be too higher in application, and one can choose a lower frequency, say $\omega_{m}=0.05$, for engineering practice. When $R V A$ is small, the time-varying delay corresponding to SSV can be approximated by [19]

$\tilde{\tau}=\tau\left(1-\frac{R V A}{2 \pi \omega_{m}}\left(\cos \left(\omega_{m} t\right)-\cos \left(\omega_{m}(t-\tau)\right)\right)\right)$.

For the intermittent control, the constant time delay, $\tau$, in Eq. (5) is replaced by

$$
\tilde{\tau}=\tau\left(1-h(\mathbf{y}) \frac{R V A}{2 \pi \omega_{m}}\left(\cos \left(\omega_{m} t\right)-\cos \left(\omega_{m}(t-\tau)\right)\right)\right),
$$



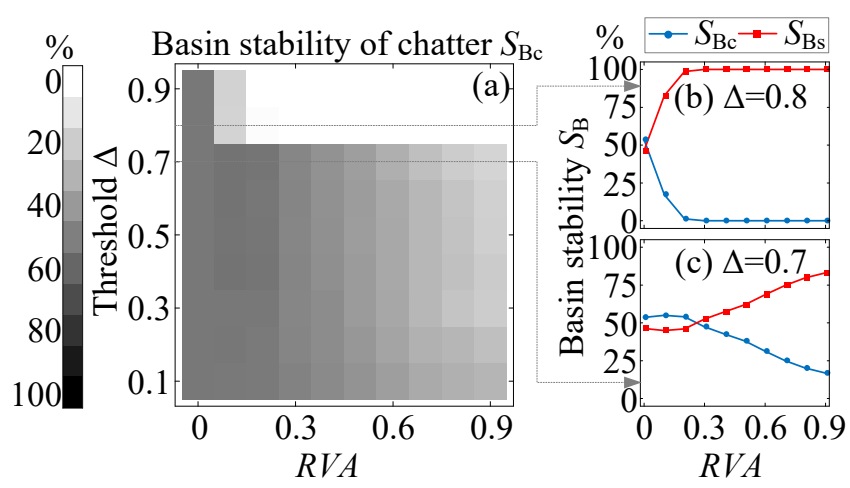

Fig. 12 (a) Effect of the state-dependent intermittent SSV on the basin stability, where the chatter occurrence for different threshold and speed varying amplitude is represented by the grayscale. Basin stabilities for $\Delta=0.8$ and 0.7 are plotted as functions of $R V A$ in Panels (b) and (c).

to make the SSV inactive out of the predefined region.

The improvement of the cutting safety induced by state-dependent intermittent SSV is displayed in Fig. 12, where the threshold and speed variation amplitude are selected for $\Delta \in[0.1,0.9]$ and $R V A \in[0,0.9]$. The greyscale distribution in Fig. 12(a) is very similar to that in Fig. 11(a), showing 0.8 as the critical threshold for cutting safety improvement. For a threshold below this value $(\Delta=0.7)$, as seen in Fig. 12(c), the cutting never be $100 \%$ safe. Moreover, $S_{\mathrm{Bc}}$ even goes up with respect to the increase of $R V A$ for $R V A<0.3$, warning us that the intermittent control may aggravate the cutting safety in the UZs. For a large threshold $(\Delta=0.8)$, Fig. 11(b) shows a significant improvement of the cutting safety, where $R V A>0.2$ is sufficient to completely eliminate the chatter orbit.

Then the effects of the three intermittent perturbations are compared for various values of $\eta_{1} / \eta_{\text {min }}$, with $\Omega$ fixed as 0.24 . Results in Figs $13(\mathrm{~b})$, (c) and (d) show much smaller UZs compared with the original UZ in Fig. 13(a). In Figs 13(b) and (c), the same threshold and feedback strength, $\Delta=0.6$ and $\epsilon=6.0$, are used, but the linear feedback performs much better than the nonlinear one, leaving only a very narrow UZ adjacent to the stability boundary. By contrast, the effect of the intermittent control with SSV for $\Delta=0.6$ and $R V A=0.6$ in Fig. 13(d) is very similar to that in Fig. 13(c), where the UZ is shrunk to be $\eta_{1} / \eta_{\min } \in[4.59,4.66]$.

\section{Conclusion}

This paper has discussed a cutting process multiple stability, which is called unsafe cutting, in a typical

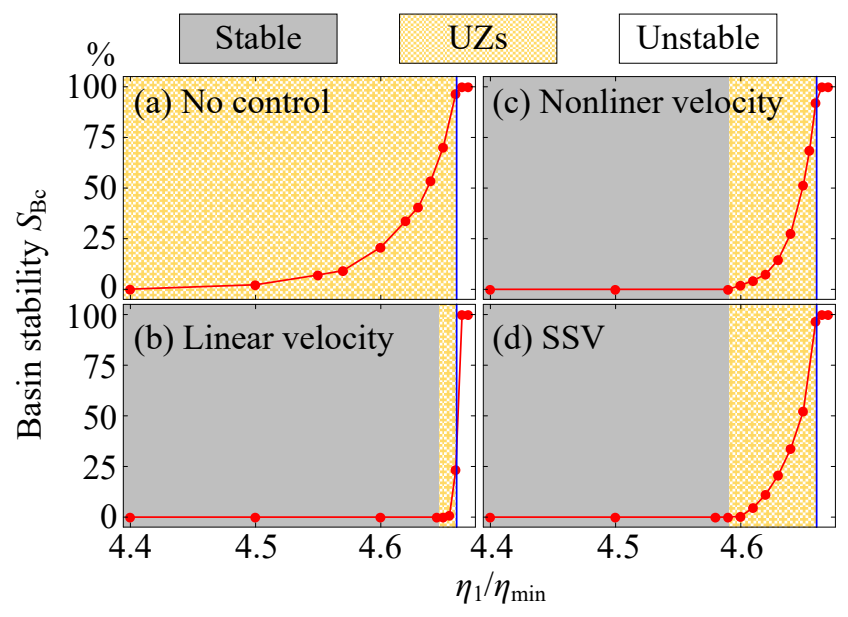

Fig. 13 The UZ in Panel (a) can be shrunk by the state-dependent intermittent control with (b) linear velocity feedback $(\Delta=0.6$ and $\epsilon=6.0)$, (c) nonlinear velocity feedback $(\Delta=0.6$ and $\epsilon=6.0)$ or $(\mathrm{d}) \operatorname{SSV}(\Delta=0.6$ and $R V A=0.6)$.

time-delayed regenerative metal cutting process. The cutting safety in the UZs was assessed based on the basin stability of chatter, i.e. the possibility of chatter occurrence. After that, the state-dependent intermittent control was introduced to perturb the unwanted chatter to improve the cutting safety, which successfully shrank the UZs for a better cutting performance.

The cutting multiple stability lacks tools to deal with its basin of attraction or basin stability due to inherent time delays introducing infinite-dimensional initial conditions. To this end, a Fourier approximation of the initial functions was proposed with its coefficients generated based on Monte Carlo principle. Then the cutting safety in the UZs was evaluated by the basin stability of chatter, $S_{\mathrm{Bc}}$, to monitor the possibility of chatter occurrence. The evolution of $S_{\mathrm{Bc}}$ with respect to the increase of $\eta_{1} / \eta_{\min }$ confirmed the "boundary layer" adjacent to the stability boundary, in which the cutting safety is significantly reduced compared with the majority of the UZs. In addition, it was revealed that the cutting safety is deteriorated by the increase of the waviness heigh in the workpiece texture, so that a smooth surface is very critical for chatter avoidance in the UZs.

Then the state-dependent intermittent control, which is active only in a predefined region to accurately destroy the chatter orbit, was introduced to improve the cutting safety. In the predefined region, three kinds of perturbation, namely linear and nonlinear velocity feedbacks and SSV, were successively employed. In general, the linear velocity 
feedback had the best performance in improving the cutting safety, which can shrink the UZ to be much narrower compared with the results of the nonlinear feedback and SSV.

The cubic nonlinear velocity feedback and SSV showed an important and similar effect that they both required a sufficient large threshold $(\Delta \geq 0.8)$ to completely destroy the UC. Below this critical threshold, the improvement of cutting safety is always limited even a very strong perturbation is used, e.g. $\epsilon=9$ or $R V A=0.9$. By contrast, only a very small perturbation is sufficient to achieve $100 \%$ safe once $\Delta \geq 0.8$. Besides, the intermittent control with SSV could deteriorate the cutting safety when a small $R V A$ is employed. However, this trend immediately disappeared when a large threshold $(\Delta \geq 0.8)$ or large variation amplitude $(R V A \geq 0.3)$ was used.

Acknowledgements This research is supported by National Natural Science Foundation of China (Grants No. 11872147, 11502048, 11772229, and 11572224), R\&D Program for International S\&T Cooperation and Exchanges of Sichuan province (Grant No. 2018HH0101) and the Fundamental Research Funds for the Central Universities (Grant No. ZYGX2018J078).

\section{Compliance with ethical standards.}

Conflict of interest. The authors declare that they have no conflict of interest.

\section{References}

1. Altintas, Y., Weck, M.: Chatter stability of metal cutting and grinding. CIRP Annals - Manufacturing Technology 53(2), 619-642 (2004). DOI 10.1016/S00078506(07)60032-8

2. Armiyoon, A.R., Wu, C.Q.: A novel method to identify boundaries of basins of attraction in a dynamical system using lyapunov exponents and monte carlo techniques. Nonlinear Dynamics 79(1), 275-293 (2015). DOI 10.1007/s11071-014-1663-z

3. Belardinelli, P., Lenci, S.: Improving the global analysis of mechanical systems via parallel computation of basins of attraction. Procedia IUTAM 22, 192-199 (2017). DOI 10.1016/j.piutam.2017.08.028

4. Dombovari, Z., Stépán, G.: On the bistable zone of milling processes. Philosophical Transactions of the Royal Society of London. Series A: Mathematical, Physical and Engineering Sciences 373(2051), 1-17 (2015). DOI 10.1098/rsta.2014.0409

5. Dombovari, Z., Wilson, R.E., Stépán, G.: Estimates of the bistable region in metal cutting. Proceedings of the Royal Society A: Mathematical, Physical and Engineering Science 464(2100), 3255-3271 (2008). DOI 10.1098/rspa.2008.0156

6. Habib, G., Kerschen, G., Stépán, G.: Chatter mitigation using the nonlinear tuned vibration absorber. International Journal of Non-Linear Mechanics 91, 103-112 (2017). DOI 10.1016/j.ijnonlinmec.2017.02.014
7. Hale, J.K.: Functional Differential Equations. Springer, Berlin (1971)

8. Höhne, K., Shirahama, H., Choe, C.U., Benner, H., Pyragas, K., Just, W.: Global properties in an experimental realization of time-delayed feedback control with an unstable control loop. Physical Review Letters 98(21), 214102 (2007). DOI 10.1103/PhysRevLett.98.214102

9. Hu, H.: Global dynamics of a duffing system with delayed velocity feedback. In: G. Rega, F. Vestroni (eds.) IUTAM Symposium on Chaotic Dynamics and Control of Systems and Processes in Mechanics, pp. 335-344. Springer Netherlands, Dordrecht (2005). DOI 10.1007/14020-3268-4_32

10. Inasaki, I., Cheng, C., Yonetsu, S.: Suppression of chatter in grinding. Bulletin of the Japan Society of Precision Engineering 9(1), 133-138 (1976)

11. Insperger, T., Barton, D.A.W., Stépán, G.: Criticality of Hopf bifurcation in state-dependent delay model of turning processes. International Journal of NonLinear Mechanics 43(2), 140-149 (2008). DOI 10.1016/j.ijnonlinmec.2007.11.002

12. Ji, J.C.: Two families of super-harmonic resonances in a time-delayed nonlinear oscillator. Journal of Sound and Vibration 349, 299-314 (2015). DOI 10.1016/j.jsv.2015.03.049

13. Kalpakjian, S., Schmid, S.R., Sekar, K.S.V.: Manufacturing Engineering and Technology, seventh edn. Pearson Education South Asia Pte Ltd, Singapore (2010)

14. Leng, S., Lin, W., Kurths, J.: Basin stability in delayed dynamics. Scientific Reports 6, 1-6 (2016). DOI $10.1038 /$ srep21449

15. Liu, Y., Chvez, J.P.: Controlling coexisting attractors of an impacting system via linear augmentation. Physica D: Nonlinear Phenomena (2017). DOI 10.1016/j.physd.2017.02.018

16. Liu, Y., Pez Chvez, J., De Sa, R., Walker, S.: Numerical and experimental studies of stickslip oscillations in drillstrings. Nonlinear Dynamics 90(4), 2959-2978 (2017). DOI 10.1007/s11071-017-3855-9

17. Liu, Y., Wiercigroch, M., Ing, J., Pavlovskaia, E.: Intermittent control of coexisting attractors. Philosophical Transactions of the Royal Society A: Mathematical, Physical and Engineering Sciences 371, 20120428 (2013). 10.1098/rsta.2012.0428

18. Long, X., Balachandran, B.: Stability analysis for milling process. Nonlinear Dynamics 49(3), 349-359 (2007). DOI 10.1007/s11071-006-9127-8

19. Long, X., Balachandran, B.: Stability of up-milling and down-milling operations with variable spindle speed. Journal of Vibration and Control 16(7-8), 1151-1168 (2010). DOI 10.1177/1077546309341131

20. Long, X., Balachandran, B., Mann, B.: Dynamics of milling processes with variable time delays. Nonlinear Dynamics 47(1), 49-63 (2007). DOI 10.1007/s11071-0069058-4

21. Long, X., Ren, S., Zheng, P.: Delayed state feedback control for milling process. Procedia IUTAM 22(Supplement C), 115-122 (2017). DOI 10.1016/j.piutam.2017.08.015

22. Molnár, T.G., Dombovari, Z., Insperger, T., Stépán, G.: On the analysis of the double Hopf bifurcation in machining processes via centre manifold reduction. Proceedings of the Royal Society A: Mathematical, Physical and Engineering Science 473, 20170502 (2017). 10.1098/rspa.2017.0502 
23. Molnár, T.G., Insperger, T., John Hogan, S., Stépán, G.: Estimation of the bistable zone for machining operations for the case of a distributed cutting-force model. Journal of Computational and Nonlinear Dynamics 11(5), 051008 (2016). DOI 10.1115/1.4032443

24. Nandakumar, K., Wiercigroch, M.: Stability analysis of a state dependent delayed, coupled two dof model of drill-string vibration. Journal of Sound and Vibration 332(10), 2575-2592 (2013). DOI $10.1016 / j . j s v .2012 .12 .020$

25. Nayfeh, A.H., Nayfeh, N.A.: Analysis of the cutting tool on a lathe. Nonlinear Dynamics 63, 395-416 (2011). DOI 10.1007/s11071-010-9811-6

26. Otto, A., Radons, G.: The influence of tangential and torsional vibrations on the stability lobes in metal cutting. Nonlinear Dynamics 82(4), 1989-2000 (2015). DOI 10.1007/s11071-015-2293-9

27. Pratt, J.R., Nayfeh, A.H.: Chatter control and stability analysis of a cantilever boring bar under regenerative cutting conditions. Philosophical Transactions of the Royal Society of London. Series A: Mathematical, Physical and Engineering Sciences 359, 759-792 (2001). DOI 10.1098/rsta.2000.0754

28. Rega, G., Lenci, S.: Dynamical integrity and control of nonlinear mechanical oscillators. Journal of Vibration and Control 14(1-2), 159-179 (2008). DOI $10.1177 / 1077546307079403$

29. Schröder, M., Mannattil, M., Dutta, D., Chakraborty, S., Timme, M.: Transient uncoupling induces synchronization. Physical Review Letters 115(5), 054101 (2015). DOI 10.1103/PhysRevLett.115.054101

30. Shang, H.: Pull-in instability of a typical electrostatic mems resonator and its control by delayed feedback. Nonlinear Dynamics 90(1), 171-183 (2017). DOI 10.1007/s11071-017-3653-4

31. Shang, H., Xu, J.: Delayed feedbacks to control the fractal erosion of safe basins in a parametrically excited system. Chaos Solitons Fract. 41(4), 1880-1896 (2009). DOI 10.1016/j.chaos.2008.07.040

32. Shi, H.M., Tobias, S.A.: Theory of finite amplitude machine tool instability. International Journal of Machine Tool Design and Research 24(1), 45-69 (1984). DOI https://doi.org/10.1016/0020-7357(84)90045-3

33. Sims, N.D.: Vibration absorbers for chatter suppression: A new analytical tuning methodology. Journal of Sound and Vibration 301(3), 592-607 (2007). DOI $10.1016 / j . j s v .2006 .10 .020$

34. Stépán, G.: Modelling nonlinear regenerative effects in metal cutting. Philosophical Transactions of the Royal Society of London. Series A: Mathematical, Physical and Engineering Sciences 359(1781), 739 -757 (2001). DOI 10.1098/rsta.2000.0753

35. Sun, X., Xu, J., Wang, F., Zhang, S.: A novel isolation structure with flexible joints for impact and ultralow-frequency excitations. International Journal of Mechanical Sciences 146-147, 366-376 (2018). DOI 10.1016/j.ijmecsci.2018.08.009

36. Sun, Y., Xiong, Z.: Modeling, analysis, and removal of chatter marks in flexible turning. The International Journal of Advanced Manufacturing Technology 93(9), 4187-4196 (2017). DOI 10.1007/s00170-017-0856-2

37. Venter, G.S., Silva, L.M.D.P., Carneiro, M.B., Da Silva, M.M.: Passive and active strategies using embedded piezoelectric layers to improve the stability limit in turning/boring operations. The International Journal of Advanced Manufacturing Technology 89(9), 2789-2801 (2017). DOI 10.1007/s00170-016-9620-2
38. Wahi, P., Chatterjee, A.: Regenerative tool chatter near a codimension 2 Hopf point using multiple scales. Nonlinear Dynamics 40(4), 323-338 (2005). DOI 10.1007/s11071-005-7292-9

39. Wang, A., Jin, W., Wang, G., Li, X.: Analysis on dynamics of a cutting tool with the thermal distortion in turning process. Nonlinear Dynamics 86(2), 1183-1191 (2016). DOI 10.1007/s11071-016-2956-1

40. Wang, H., Hu, H., Wang, Z.: Global dynamics of a duffing oscillator with delayed displacement feedback. International Journal of Bifurcation and Chaos 14(08), 2753-2775 (2004). DOI 10.1142/S0218127404010990

41. Wang, Q., He, Y., Tan, G., Wu, M.: State-dependent intermittent control of non-linear systems. IET Control Theory \& Applications 11(12), 1884-1893 (2017). DOI 10.1049/iet-cta.2016.1385

42. Wiercigroch, M., Budak, E.: Sources of nonlinearities, chatter generation and suppression in metal cutting. Philosophical Transactions of the Royal Society of London. Series A: Mathematical, Physical and Engineering Sciences 359(1781), 663-693 (2001). DOI $10.1098 /$ rsta.2000.0750

43. Wiercigroch, M., Nandakumar, K., Pei, L., Kapitaniak, M., Vaziri, V.: State dependent delayed drillstring vibration: theory, experiments and new model. Procedia IUTAM 22, 39-50 (2017). DOI https://doi.org/10.1016/j.piutam.2017.08.007

44. Xu, J., Lu, Q., Huang, K.: Controlling erosion of safe basin in nonlinear parametrically excited systems. Acta Mechanica Sinica 12(3), 281-288 (1996). DOI 10.1007/BF02486814

45. Yadav, K., Kamal, N.K., Shrimali, M.D.: Intermittent feedback induces attractor selection. Physical Review E 95(4), 042215 (2017). DOI 10.1103/PhysRevE.95.042215

46. Yan, Y., Xu, J.: Suppression of regenerative chatter in a plunge-grinding process by spindle speed. ASME Journal of Manufacturing Science and Engineering 135(4), 041019-041019 (2013). 10.1115/1.4023724 doi: $10.1115 / 1.4023724$

47. Yan, Y., Xu, J., Wang, W.: Nonlinear chatter with large amplitude in a cylindrical plunge grinding process. Nonlinear Dynamics 69(4), 1781-1793 (2012). DOI 10.1007/s11071-012-0385-3

48. Yan, Y., Xu, J., Wiercigroch, M.: Non-linear analysis and quench control of chatter in plunge grinding. International Journal of Non-Linear Mechanics 70, 134144 (2015). DOI 10.1016/j.ijnonlinmec.2014.06.012

49. Yan, Y., Xu, J., Wiercigroch, M.: Regenerative and frictional chatter in plunge grinding. Nonlinear Dynamics 86(1), 283-307 (2016). DOI 10.1007/s11071-016-2889-8

50. Yan, Y., Xu, J., Wiercigroch, M.: Regenerative chatter in self-interrupted plunge grinding. Meccanica 51(12), 3185-3202 (2016). DOI 10.1007/s11012-016-0554-4

51. Yan, Y., Xu, J., Wiercigroch, M.: Basins of attraction of the bistable region of time-delayed cutting dynamics. Physical Review E 96(3), 032205 (2017). DOI 10.1103/PhysRevE.96.032205

52. Yan, Y., Xu, J., Wiercigroch, M.: Stability and dynamics of parallel plunge grinding. The International Journal of Advanced Manufacturing Technology 99(1), 881-895 (2018). DOI 10.1007/s00170-018-2440-9

53. Zhang, S., Xu, J., Chung, K.W.: Desynchronizationbased congestion suppression for a star-type internet system with arbitrary dimension. Neurocomputing (2017). DOI 10.1016/j.neucom.2017.05.023 
54. Zheng, Y.G., Sun, J.Q.: Attractive domain of nonlinear systems with time-delayed feedback control and timedelay effects. Procedia IUTAM 22, 51-58 (2017). DOI https://doi.org/10.1016/j.piutam.2017.08.008 